\title{
Climatología básica de tormentas en España
}

https://doi.org/10.31978/639-19-010-0.247

\author{
José Ángel Núñez Mora' ${ }^{1}$ (jnunezm@aemet.es) \\ Manuel Antonio Mora García² (mmoray@aemet.es) \\ Jesús Riesco Martín ${ }^{3}$ (jriescom@aemet.es) \\ ${ }^{1}$ AEMET / Delegación Territorial en la Comunidad Valenciana \\ ${ }^{2}$ AEMET / Delegación Territorial en Castilla y León \\ ${ }^{3}$ AEMET / Delegación Territorial en Andalucía, Ceuta y Melilla / Centro Meteorológico de Málaga
}

\begin{abstract}
RESUMEN
La climatología de descargas eléctricas y tormentas en España, tanto en territorio terrestre como en el entorno marítimo próximo, constituye una importante referencia a la hora de abordar predicciones operativas referidas a tormentas. En este trabajo se pone de manifiesto una climatología básica de descargas eléctricas y días de tormenta en un periodo reciente de 10 años (2007-2016) a partir de la información contenida en la base de datos de la red de descargas de AEMET en España (Península, Baleares y Canarias).

La climatología descriptiva de las descargas eléctricas y de días de tormenta se realiza mediante gráficos y mapas de densidad espacial referentes a distribuciones mensuales, estacionales, anuales y horarias de descargas eléctricas, diferenciando su polaridad (negativa o positiva). Finalmente, como resultado del análisis espaciotemporal de la distribución de tormentas, se extraen conclusiones básicas acerca de la climatología de las tormentas así como de los patrones básicos observados, que servirán de ayuda a los predictores operativos en sus tareas de vigilancia y predicción de tormentas en sus zonas de responsabilidad.
\end{abstract}

PALABRAS CLAVE: atlas; tormentas; descargas eléctricas; climatología de tormentas.

\section{INTRODUCCIÓN}

La actividad eléctrica asociada a las tormentas es un fenómeno meteorológico de gran impacto, que causa numerosas pérdidas de vidas humanas y cuantiosos daños materiales. Por tanto, conocer la distribución espacial y temporal de las descargas eléctricas en tiempo real es fundamental para la adecuada vigilancia de este fenómeno meteorológico adverso, que afecta notablemente a las actividades humanas realizadas al aire libre, a la gestión del tráfico aéreo y las operaciones de repostaje de aeronaves, provoca averías e interrupciones de suministro eléctrico y daños en aerogeneradores de parques eólicos y otras infraestructuras, genera incendios forestales, etc. Los datos almacenados a lo largo de los años también son fundamentales para una mejor comprensión de la actividad tormentosa, así las climatologías de descargas eléctricas y de número de días de tormenta contribuyen a una mejor predicción de este fenómeno. Asimismo, estos datos son imprescindibles para la investigación de determinados siniestros, siendo demandados por las compañías aseguradoras y juzgados.

Existen distintos sistemas de detección de rayos ubicados en tierra, cuyas características permiten detectar descargas nube a tierra, nube a nube o intranube, o ambos tipos. El sistema empleado por AEMET pertenece a los conocidos como de alcance medio o de precisión, cuyos sensores están espaciados a distancias inferiores 
a 400 km, y por tanto son idóneos para regiones como la península ibérica. Estos sensores están diseñados para detectar las emisiones en baja frecuencia (LF). En estas frecuencias, la señal electromagnética generada por la descarga es intensa y además su propagación por la superficie terrestre sufre poca atenuación.

La probabilidad de detección de rayos nube a tierra de este tipo de redes oscila entre un $85 \%$ y un $95 \%$, decayendo a valores entre un $70 \%$ y un $90 \%$ para las descargas que componen un rayo (estas diferencias se deben a la multiplicidad del rayo, ya que basta una sola descarga de las múltiples asociadas al rayo para detectarlo).

La red de detección de rayos (REDRA) de AEMET está compuesta por tres subsistemas encargados respectivamente de la detección de descargas eléctricas (SDDE), el control de los equipos, el análisis y localización de sus medidas (SCAL) y el almacenamiento y la explotación de estos datos (CATS). El SDDE está formado por veinte equipos detectores de descargas eléctricas distribuidos por el territorio peninsular (14) y los archipiélagos balear (1) y canario (5).

Además de los equipos de la red de AEMET, REDRA recibe datos de una red de cuatro equipos de detección de Portugal, para cubrir de forma óptima toda la península ibérica y los mares circundantes (figura 1). Así mismo, en virtud de un acuerdo de cooperación con el Servicio Meteorológico francés, AEMET también recibe información proveniente de catorce equipos de la red francesa, dos de la red italiana y cinco de la red marroquí.

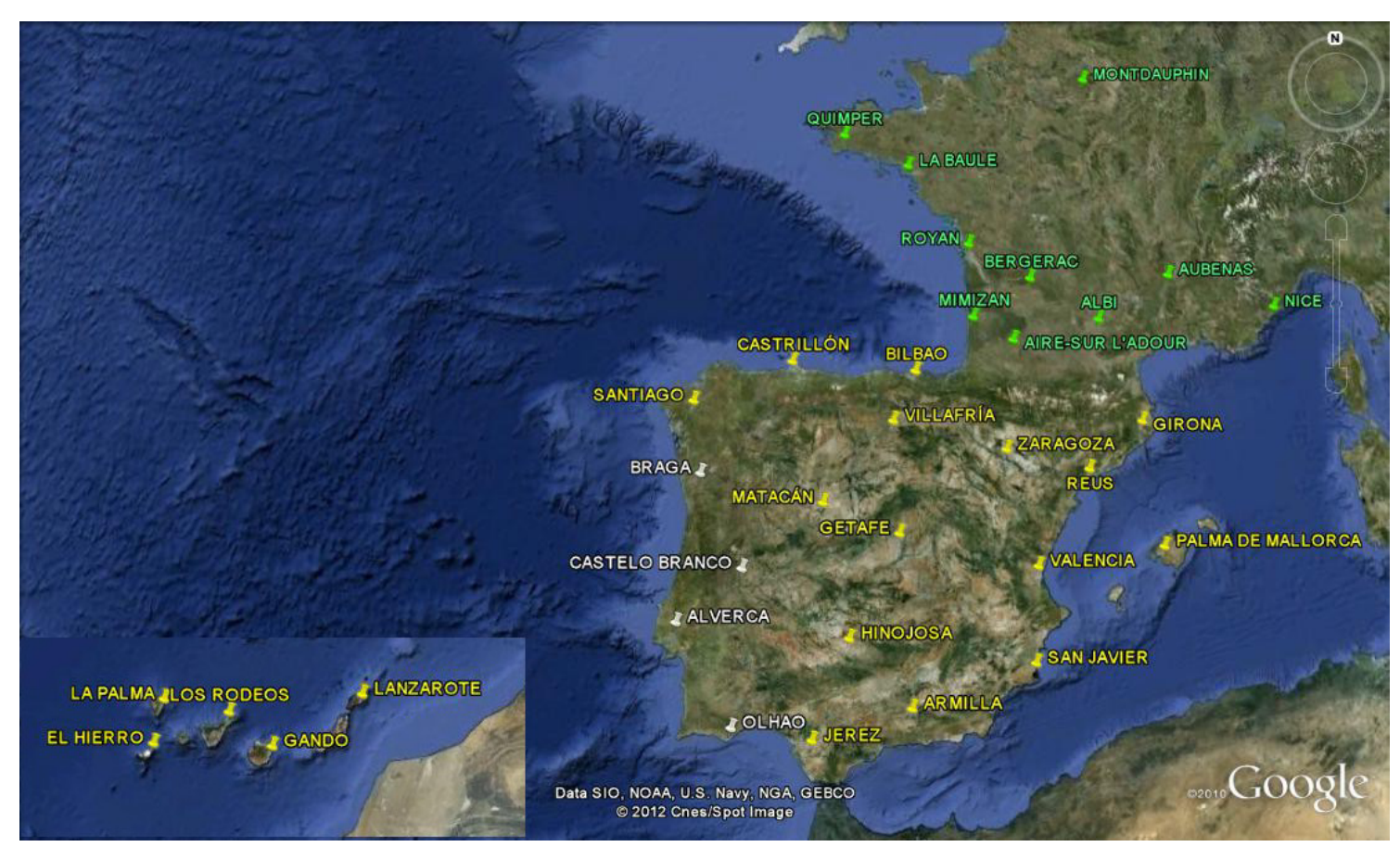

Figura 1. Localización de los sensores de detección de rayos de las diferentes redes. Amarillo: AEMET, blanco: Instituto de Meteorología de Portugal, verde: Météo-France.

\section{SELECCIÓN DE DATOS. PARÁMETROS DE CALIDAD Y PRECISIÓN DE LA RED. FILTRADO DE DATOS}

Los datos proceden inicialmente de dos áreas representativas (una para la Península y Baleares y otra para Canarias). El entorno de la Península y Baleares preseleccionado es el limitado por los paralelos $45^{\circ} \mathrm{N}$ y $34^{\circ} \mathrm{N}$ y los meridianos $11^{\circ} \mathrm{W}$ y $5^{\circ} \mathrm{E}$, que abarca $2181000 \mathrm{~km}^{2}$. En principio se seleccionaron 17 años de datos, entre 2000 y 2016, con lo que se obtuvieron un total de 25528942 registros. 
Para la zona de las islas Canarias se ha preseleccionado el área comprendida entre los paralelos $30^{\circ} \mathrm{N} \mathrm{y}$ $27^{\circ} \mathrm{N}$ y los meridianos $19^{\circ} \mathrm{W}$ y $12^{\circ} \mathrm{W}$, que abarca una superficie de $260232 \mathrm{~km}^{2}$, aproximadamente ocho veces y media inferior a la ventana geográfica de la Península e islas Baleares. La red canaria se instaló después que la de la Península, y los datos con los que se ha trabajado son de un periodo de 10 años (2007-2016) con un total de 178247 registros.

Las características que definen una red de detección de rayos son la probabilidad de detección de descargas o rayos y la precisión en la localización de las mismas. Ambos valores van a depender de la posición relativa de la descarga respecto a los detectores, del número de sensores que detectan la descarga y de la intensidad de la misma. Respecto a la red de detección de descargas eléctricas de AEMET se estima que actualmente la precisión en la localización es de unos 500 m y que la eficiencia de detección es del $90 \%$.

La precisión en la localización está muy relacionada con el valor del semieje mayor de la elipse de confianza (a menor valor del semieje mayor medio de las descargas, la red será más precisa). Otro parámetro utilizado para determinar la calidad de los datos individuales viene definido por el parámetro $\chi^{2}$ (también denotado como «chi-cuadrado», o «chi-2»), que consiste en una medida normalizada del «grado de acuerdo»o «consistencia» entre todos los sensores que intervienen en la localización de una descarga. Idealmente, esta distribución estadística debería tener un valor medio y mediana de 1, pero en la realidad, debido a los errores angulares y temporales de los diferentes sensores que intervienen, esto no sucede así. Según la bibliografía consultada, valores individuales entre 0 y 3 se consideran buenos, y entre 3 y 10 aceptables.

Una vez obtenidos todos los datos, se ha procedido a filtrar y eliminar aquellos que tienen un parámetro chi-cuadrado superior o igual a 10, ya que se consideran datos erróneos (probablemente por sensores mal calibrados, ruidos inusuales o errores en el ángulo de posicionamiento de la descarga o en la hora de su detección). En total, se han eliminado 420718 registros de la Península y Baleares lo que supone el 1,65\% del total, aunque el número de descargas consideradas erróneas (chi-cuadrado mayor o igual a 10) ha ido descendiendo en el tiempo, de forma que a partir del año 2007 el porcentaje de registros erróneos es inferior al $1 \%$ del total anual. De los registros de las islas Canarias se han eliminado 1543 lo que supone el 0,87\% del total, con lo que la base de datos final de Canarias cuenta con un total de 176704 registros.

Con la base de datos ya depurada, se ha procedido a la interpolación espacial de los dos parámetros de calidad. Para la Península e islas Baleares se ve un claro descenso con el tiempo de ambos parámetros, con una tendencia a la estabilización a partir de 2007, que es el año de inicio de las estadísticas para el caso de las islas Canarias. A partir de 2007, el $\chi^{2}$ medio anual está estabilizado en valores próximos a 1,2, y el semieje mayor queda estabilizado alrededor de $2 \mathrm{~km}$. Esta tendencia observada puede estar relacionada con las progresivas mejoras que se han ido introduciendo en la red, las mejoras en el filtrado de datos erróneos o sospechosos, y sobre todo con la integración de las redes ibéricas, con la inclusión de los datos registrados por Portugal, y la instalación de la red de Canarias. Como resultado, se ha mejorado la precisión de la red en el suroeste de la zona de estudio peninsular.

Para las islas Canarias, desde la puesta en marcha de la red, los parámetros de calidad están muy estables durante todo el periodo, aunque se ve una mejora en la precisión de la localización a partir de 2015, cuando desciende de valores medios anuales de $8 \mathrm{~km}$ a valores medios anuales de $4 \mathrm{~km}$. Desde 2007, el chi- 2 medio anual está estabilizado en valores próximos a 1,1.

Con los resultados obtenidos de la fase de control de calidad y filtrado de datos, se ha optado por la elección del periodo 2007-2016 como base de datos, y prescindir de las descargas anteriores a 2007 en el caso de la Península y Baleares. Este periodo es suficientemente homogéneo para trabajar con garantías, además de que coincide con el periodo de datos para el caso de las islas Canarias, por lo que con estos criterios se puede construir una climatologías de descargas y tormentas homogéneo para las dos redes, la de la Península e islas Baleares y la de las islas Canarias, de forma que se pueden construir mapas y gráficos que permitirán comparar la distribución de descargas y tormentas en ambos territorios. 
Además, para el caso de la Península y Baleares, es aconsejable también reducir la extensión de la rejilla de datos a emplear, ya que la zona de los bordes norte, oeste y sur perdía calidad con respecto al resto de la zona geográfica inicialmente elegida. La ventana seleccionada abarca una extensión que comprende las zonas terrestres de la Península, Ceuta, Melilla y Baleares, por lo que se ha reducido la zona inicial de estudio en un $1^{\circ}$ por el norte, oeste y sur, quedando igual por el este. Respecto a la ventana geográfica inicial de Canarias no se ha modificado.

En definitiva, los datos finales con los que se ha trabajado responden a las siguientes características:

1. Descargas nube-tierra filtradas (datos de la base de datos de AEMET con $\left.\chi^{2}<10\right)$.

2. Periodo 2007-2016.

3. Ventanas geográficas (figura 2):

- Península-Baleares: $44^{\circ} \mathrm{N}$ a $35^{\circ} \mathrm{N}$ y $10^{\circ} \mathrm{W}$ a $5^{\circ} \mathrm{E}$

- Canarias: paralelos $30^{\circ} \mathrm{N} \mathrm{a} 27^{\circ} \mathrm{N}$ y $19^{\circ} \mathrm{W}$ a $12^{\circ} \mathrm{W}$.

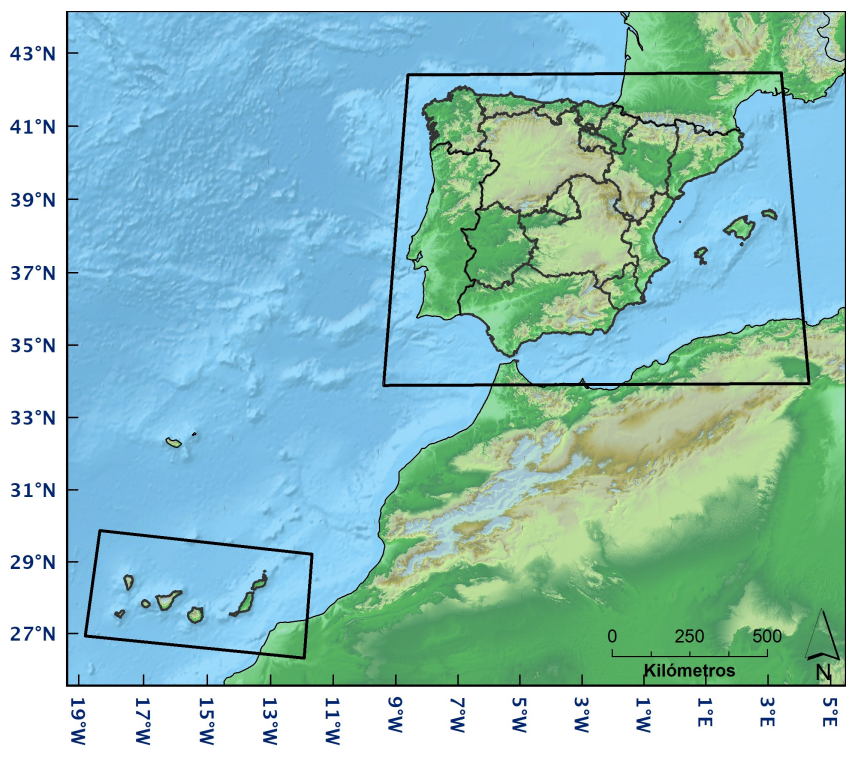

Figura 2. Ventanas geográficas utilizadas para realizar el estudio estadístico y representar distintos mapas.

\section{ESTUDIO ESTADÍSTICO DESCRIPTIVO Y MAPAS DE DENSIDAD DE DESCARGAS ELÉCTRICAS}

En la base de datos depurada de descargas del periodo 2007-2016 hay un total de 12577044 registros en la ventana geográfica de la Península e islas Baleares. En los diez años del estudio, el de mayor actividad fue 2014, y el de menor actividad 2012, como puede apreciarse en la figura 3.

En Canarias se registran muchos menos rayos que en la mayoría de zonas peninsulares. En el periodo de 10 años estudiados (2007-2016) se dispone de un total de 176704 registros. La superficie considerada para el caso de Canarias es casi ocho veces y media inferior a la de la ventana geográfica de estudio de la Península y Baleares, pero en valor absoluto, el número total de descargas es 72 veces inferior en Canarias que en la Península y Baleares. El escaso número de tormentas en Canarias, que además se distribuyen irregularmente en el tiempo, da lugar a que exista fuerte contraste interanual, como se observa en la figura 4.

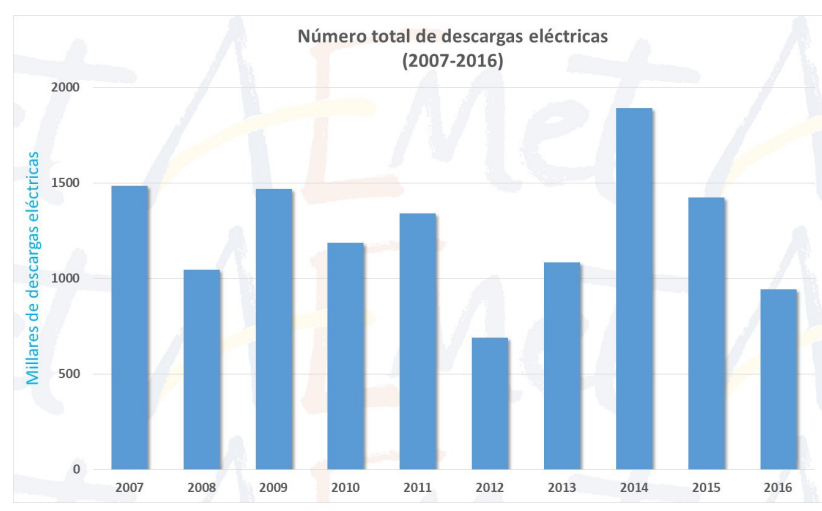

Figura 3. Número total anual de descargas eléctricas en la Península y Baleares (millares de descargas).

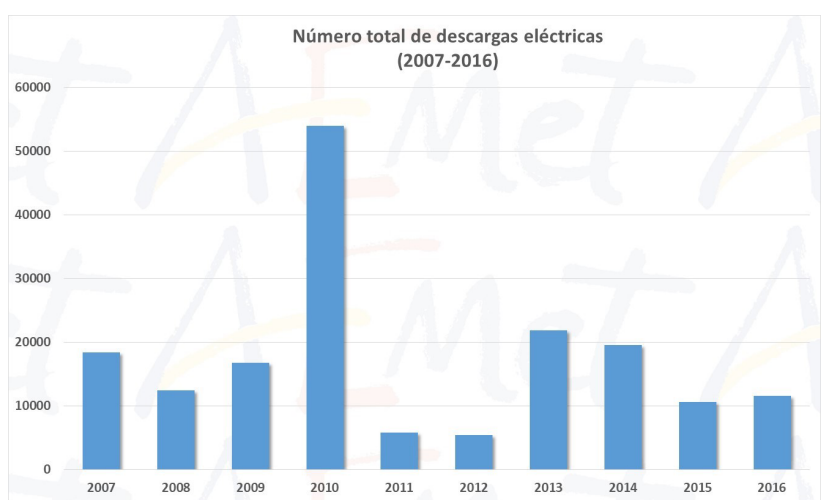

Figura 4. Número total anual de descargas eléctricas en las islas Canarias. 


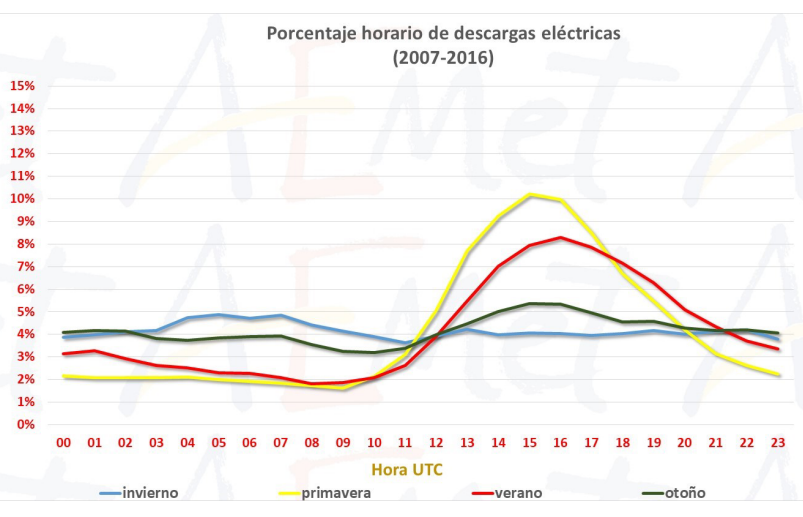

Figura 5. Porcentaje horario de descargas eléctricas por estaciones en la Península y Baleares.

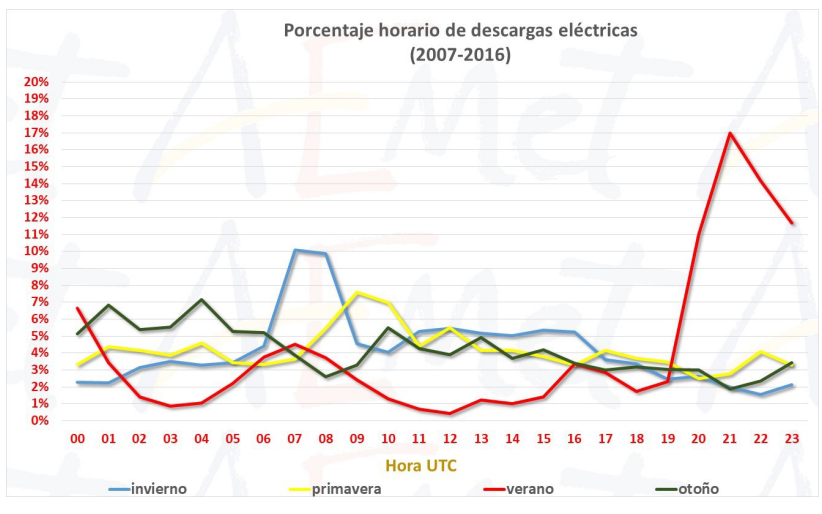

Figura 6. Porcentaje horario estacional de descargas eléctricas en las islas Canarias.

En primavera y verano hay un clarísimo predominio en la Península y Baleares de las tormentas de primera hora de la tarde (ligeramente más adelantadas en horario las tormentas primaverales), lo que indica que en primavera y verano las tormentas se desarrollan en fase con el calentamiento diurno y con las convergencias de viento que surgen en el interior de la Península debido al distinto comportamiento térmico de las tierras y los mares a partir de mediodía. En primavera y verano el porcentaje de descargas respecto al total estacional en horario nocturno y por la mañana es mucho más bajo que el porcentaje de descargas en esa fase del día en otoño e invierno (figura 5).

Sin una clara preferencia horaria se presenta la distribución de las descargas en las tormentas de invierno y de otoño, aunque en invierno se suele observar una ligera mayor frecuencia de madrugada y en otoño por la tarde. Sin embargo en ambos casos el mecanismo de disparo convectivo responde a situaciones en las que aparece una depresión o vaguada atlántica con pasos frontales, o bien la existencia de una dana (depresión aislada de niveles altos), respondiendo más a factores dinámicos que al calentamiento diurno.

En Canarias no se obtiene un patrón tan claro como en la Península y Baleares. Aunque hay un máximo muy destacado en verano a última hora del día entre las 20:00 y las 23:59 (de 21:00 a 00:59 hora oficial insular de verano), hay que tener en cuenta que junio y julio, que son meses veraniegos, apenas registran tormentas en Canarias, y solo agosto es un mes con un número de descargas suficiente para afirmar que existe un patrón de máximo de descargas en las islas Canarias a última hora del día (figura 6).

La zona de mayor densidad de descargas en la Península se encuentra en el Maestrazgo turolense y castellonense (figura 7), entre las localidades de Mosqueruela, Valdelinares y la Iglesuela del Cid (Teruel), y Vilafranca

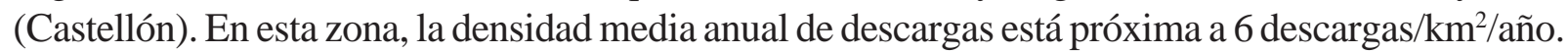

Figura 7.

Densidad anual de descargas en la

Península e islas Baleares (descargas $\left./ \mathrm{km}^{2}\right)$.
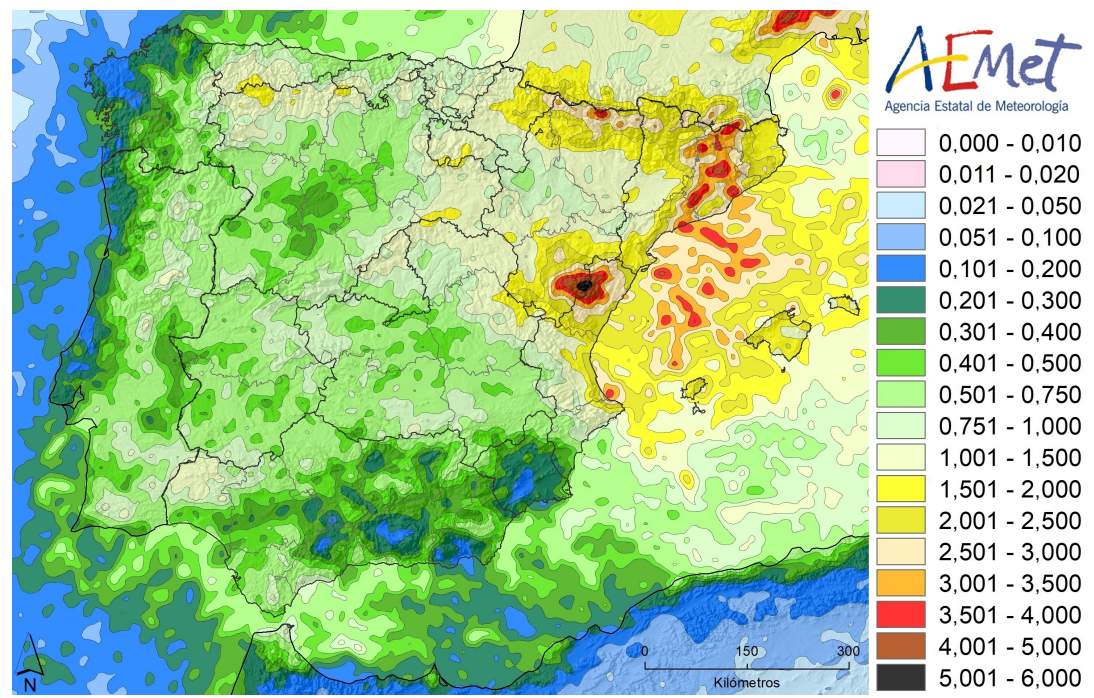


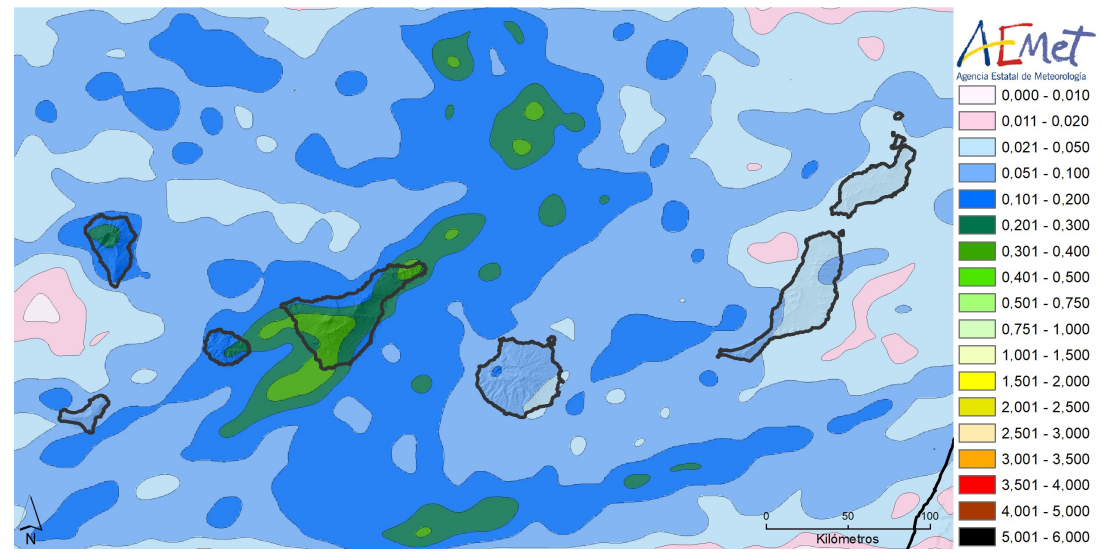

Figura 8. Densidad anual de descargas en las islas Canarias (descargas $/ \mathrm{km}^{2}$ ).

El realce orográfico como factor desencadenante de tormentas en primavera y verano, así como la convergencia de vientos hace de esta zona la de mayor actividad ceráunica de España. La zona de mínimos en la

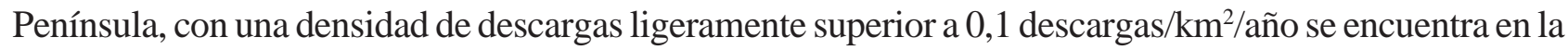
provincia de A Coruña.

En Canarias la mayor actividad se presenta en el otoño e invierno y la zona de mayor densidad abarca gran parte de la isla de Tenerife y la isla de La Palma (figura 8), con menor actividad convectiva en las islas orientales.

\section{DÍAS DE TORMENTA}

La Organización Meteorológica Mundial estableció en 1956 la definición de día de tormenta considerando que ha habido tormenta en un observatorio cuando en un día natural (civil) se ha oído al menos un trueno. Por tanto, un día de tormenta es registrado como tal independientemente del número real de tormentas que haya habido ese día en el observatorio. Cuando se ve el relámpago pero no se oye el trueno, no se considera que ha habido tormenta en el observatorio, y cuando la tormenta comienza antes de la medianoche y termina después de la medianoche, se computa como dos días de tormenta distintos.

Con este criterio, la consideración de día de tormenta es independiente de la intensidad y duración de las mismas, y lo más importante para el objeto de este trabajo: no se establece un círculo de radio fijo dentro del cual se considera de forma objetiva que ha habido tormenta. La misma Organización Meteorológica Mundial indica que el requisito de que se oiga realmente el trueno, limita el área cubierta por cada punto de observación a un círculo con un radio de unos $20 \mathrm{~km}$, sin más precisión. El primer objetivo para trazar una cartografía de número de días de tormenta a partir de los datos de la red de detección descargas, es tratar de definir de forma objetiva la distancia a un punto (que podría ser un observatorio meteorológico) desde la que se oye el trueno.

Los estudios de la audibilidad del trueno sugieren que estos se escuchaban a distancias de 8 y $19 \mathrm{~km}$ (Changnon, 1989). Otros autores establecen otros valores: entre $15 \mathrm{~km}$ y $20 \mathrm{~km}$ (Finke y Hauf, 1996); 11,3 km (MäKelä, EnNo y HaApalainen, 2014) en el caso de los países nórdicos indicando que para su entorno correlaciona bien con los datos convencionales basados en observaciones humanas, o un valor medio de 17,5 km (CZERNECKI, TASZAREK, KolEndowicz y KonARSKI, 2016) en un estudio que relacionaba observaciones de días de tormenta contenidas en informes SYNOP y los datos de la red de detección de descargas de Polonia.

En España, se ha trabajado con radios de $10 \mathrm{~km}$ con resultados satisfactorios (Pérez Puebla, ZancAJo Rodríguez y GonZÁlez MárqueZ, 2007), e incluso valores menores como entre 5 y 6 km (SANZ y otros, 
2016). En Galicia se obtuvo un círculo de $12 \mathrm{~km}$ de radio como el más adecuado para los observatorios de la comunidad autónoma gallega, para lo que cruzaron datos de observación convencional con datos de la red de descargas (GómeZ ViÑas, 2016).

Al igual que se ha hecho en varios de los estudios citados anteriormente, para establecer el radio medio del círculo dentro del cual se notifican tormentas en España, se han correlacionado los datos de las observaciones convencionales que están anotadas en el banco de datos climatológico nacional de AEMET del periodo 2007-2016 (el mismo periodo que el de la base de datos de descargas empleada en esta climatología), con los datos de la red de descargas eléctricas.

Para realizar esta correlación, se han seleccionado los datos de los observatorios de la red principal de AEMET, y se les ha sometido a un control de calidad, ya que no todos los observatorios disponen de personal en horario de 24 horas o durante los 365 días del año para cifrar meteoros, y aunque muchos de ellos, sobre todo en capitales de provincia medianas y pequeñas, a pesar de tener un horario inferior a 24 horas, siguen prestando atención a los meteoros que se producen fuera de su horario de trabajo para anotarlos al día siguiente, otros, sobre todo situados en grandes ciudades, no lo hacen. Además, se han completado los datos de los observatorios de la red principal de AEMET con datos de la red termopluviométrica secundaria que cifraron meteoros entre el periodo 2007-2016 sin presentar ninguna laguna. Los datos de estos observatorios se consideran muy importantes, ya que básicamente proceden de zonas rurales no cubiertas por observatorios con personal propio de AEMET. Son estaciones gestionadas por observadores muy expertos y comprometidos, que ofrecen garantía de observación continua de meteoros. Además, los datos de estos observatorios se han sometido a un control de calidad, y se ha procurado introducir datos de observatorios de calidad contrastada situados en zonas singulares con gran densidad de descargas.

Para el caso de Canarias, la tarea de establecer un radio alrededor del cual se notifican tormentas es más compleja que para la Península. Primero porque es un área más pequeña, lo que dificulta la posibilidad de disponer de un número suficiente de observatorios de calidad contrastada que cifren meteoros y, en concreto, que cifren la existencia o no de tormenta durante las 24 horas del día. Otra dificultad añadida para el caso de Canarias es que, al igual que ocurría con las descargas, hay muy pocas tormentas, lo que también complica la tarea de hacer una buena correlación entre datos manuales basados en observaciones humanas y datos automáticos basados en los registros de la red de descargas, sobre todo debido a que en una población reducida de datos, una estadística de 10 años puede ser insuficiente.

Como era de esperar, se han obtenidos resultados muy diferentes entre unos observatorios y otros, ya que la observación y notificación de tormenta por observadores está sometida a múltiples incertidumbres, como son las condiciones de audibilidad del observatorio, las condiciones atmosféricas que condicionan la propagación del sonido, la situación geográfica del observatorio, y muchos otros factores subjetivos. Todos estos condicionantes indican las ventajas de emplear la red de descargas eléctricas para realizar una cartografía de días de tormenta, ya que una vez establecido el radio de búsqueda de descargas alrededor de cada punto del territorio, queda establecido un criterio uniforme y objetivo para la definición de día de tormenta en todo el territorio, criterio que además está calculado teniendo en cuenta las observaciones convencionales realizadas de acuerdo a los criterios de la Organización Meteorológica Mundial.

Teniendo en cuenta todo lo expuesto anteriormente, el promedio de la distancia calculada para la notificación de tormenta en la superficie del territorio de la Península y Baleares es de 10,1 km, y para Canarias es de $8,9 \mathrm{~km}$, inferior al de la Península. Estos son, por tanto, los valores que se utilizaron para realizar de forma objetiva la cartografía de número de días de tormenta en España.

En el periodo 2007-2016, la zona con más días de tormenta en la Península e islas Baleares (máximo) se encuentra al sur del macizo de Cotiella, entre las comarcas del Sobrarbe y la Ribagorza, en la provincia de Huesca (figura 9). En esos 10 años, en una zona del valle de la Fueva se registraron 379 días de tormenta, con un promedio por tanto de 37,9 días de tormenta al año. Asimismo en el área entre los municipios de 


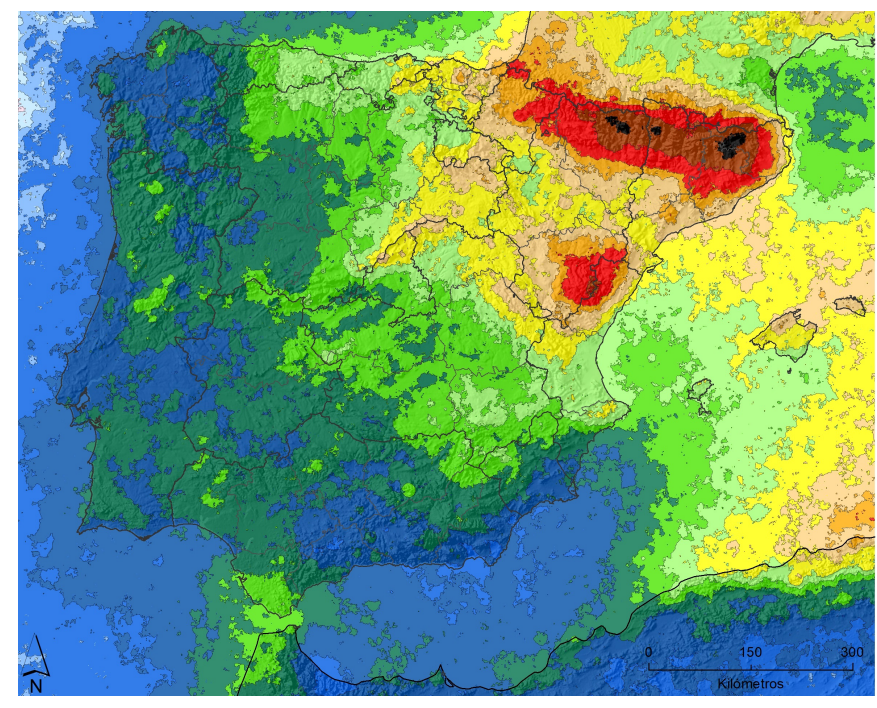

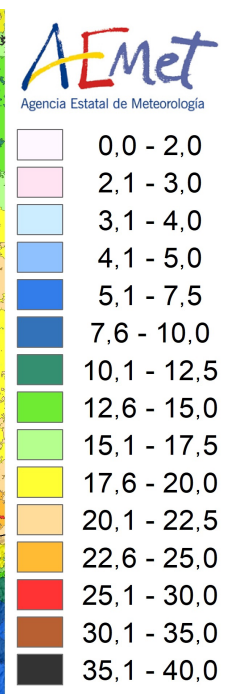

Figura 9.

Número medio anual de días de tormenta en la Península e islas Baleares.

Campo y Fanlo, pertenecientes a las citadas comarcas oscenses, el promedio anual supera los 35 días de tormenta.

En otras zonas del norte de Cataluña también existen áreas con un promedio anual superior a 35 días de tormenta al año. Se trata de las comarcas del Pallars Sobirà y de la Alta Ribagorça en la provincia de Lleida, de la comarca del Ripollès en el noroeste de la provincia de Girona y de las comarcas de Osona y el Alt Berguedà al norte de la provincia de Barcelona.

También es reseñable el hecho de que se registran muchos más días de tormenta en la parte española de los Pirineos, al sur de la cordillera, que en la parte francesa, probablemente por encontrarse en la zona de barlovento asociado a los flujos de las situaciones tormentosas.

Fuera de esta zona al sur de los Pirineos orientales, solo en el Maestrazgo turolense y Maestrat castellonense, entre Cantavieja, Mosqueruela, Vilafranca y Vistabella del Maestrat, se superan los 30 días de tormenta al año en el promedio 2007-2016. Esta zona coincide además con la de mayor densidad de descargas en España, es decir, hay más tormentas en la vertiente sur de los Pirineos orientales, pero las tormentas del Maestrazgo registran mayor cantidad de rayos.

El mínimo anual de días de tormenta en la Península y Baleares se registra en la costa mediterránea de Andalucía oriental, entre Motril, en Granada, y Vera, en Almería, donde hay entre 6 y 7 días de tormenta al año.

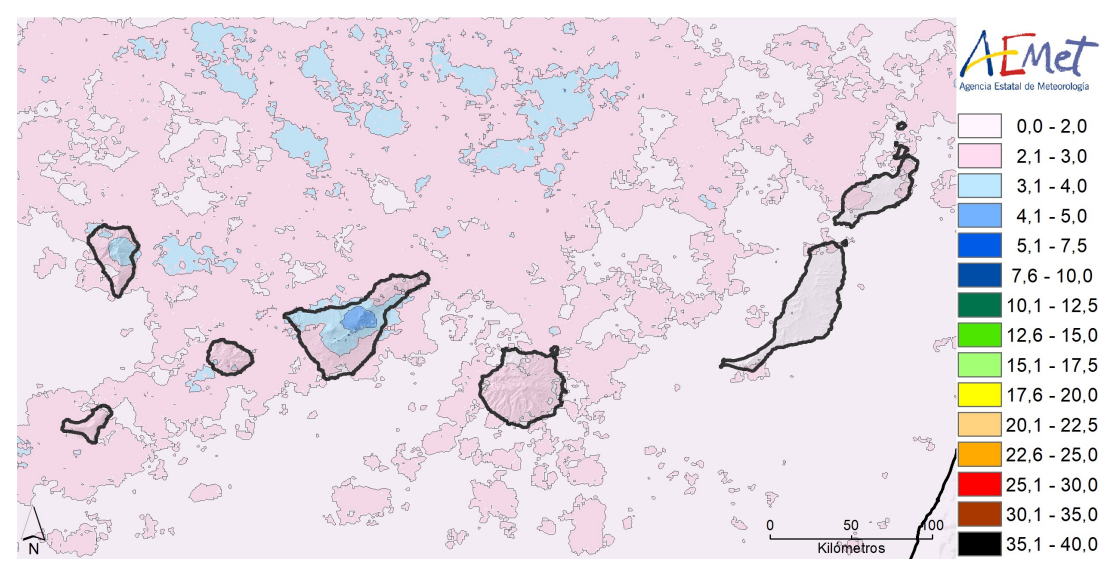

Figura 10. Número medio anual de días de tormenta en Canarias. 
En Canarias, al igual que ocurría con la densidad de descargas, hay muchos menos días de tormenta que en la Península y Baleares. Por ejemplo las dos capitales canarias tienen menos de la mitad de días de tormenta que la capital de la Península que presenta la cantidad más baja (Almería). La zona de mayor número de días de tormenta en Canarias se sitúa en la isla de Tenerife, en zonas altas y medianías al nordeste del Teide, donde hay entre 4 y 5 días de tormenta de media al año (figura 10). En otras zonas próximas de la isla de Tenerife y zonas altas de La Palma hay entre 3 y 4 días de tormenta de media al año. Los valores mínimos se registran en las islas orientales.

Por estaciones, aunque en número escaso (3-4 días anuales), se observa una mayor frecuencia de tormentas invernales (figura 11, arriba izquierda) en el área cantábrica debido a las advecciones frías del norte y en el litoral atlántico debido al paso de frentes atlánticos, así como el área mediterránea asociado a vaguadas en niveles medios, en general por el contraste térmico entre el aire frío invernal y el mar relativamente cálido, con acusado efecto de la continentalidad (prácticamente no se producen tormentas en el interior peninsular).

En primavera (figura 11, arriba derecha) oscila entre 6-8 días en el nordeste montañoso y 3-5 días en ambas mesetas y cornisa cantábrica (efecto conjunto del mayor caldeamiento solar y la orografía como mecanismo de disparo de las tormentas), con mínimos en el extremo NW y en el SE; entre 1 y 2 días. En verano (figura 11, abajo izquierda) destacan los dos máximos de hasta 20 días en Pirineos y el sistema Ibérico, con valores en el resto del cuadrante NE superiores a 8 días. Se observa un fuerte gradiente SW-NE, con mínimos (en torno a 1 día de tormenta o menos) en el litoral atlántico y SE, como consecuencia de las tormentas de masa de aire habituales en el periodo veraniego en el interior peninsular, debidas al caldeamiento solar y la orografía como mecanismo de disparo, al margen de los episodios de tormentas originados por el paso de vaguadas atlánticas. En otoño (figura 11, abajo derecha) el máximo se encuentra en el área de las islas Baleares y el Mediterráneo oriental, con valores entre 12 y 14 días, valores un poco inferiores en las zonas próximas mediterráneas y el área pirenaica, el mínimo en el cuadrante noroccidental, con valores entre
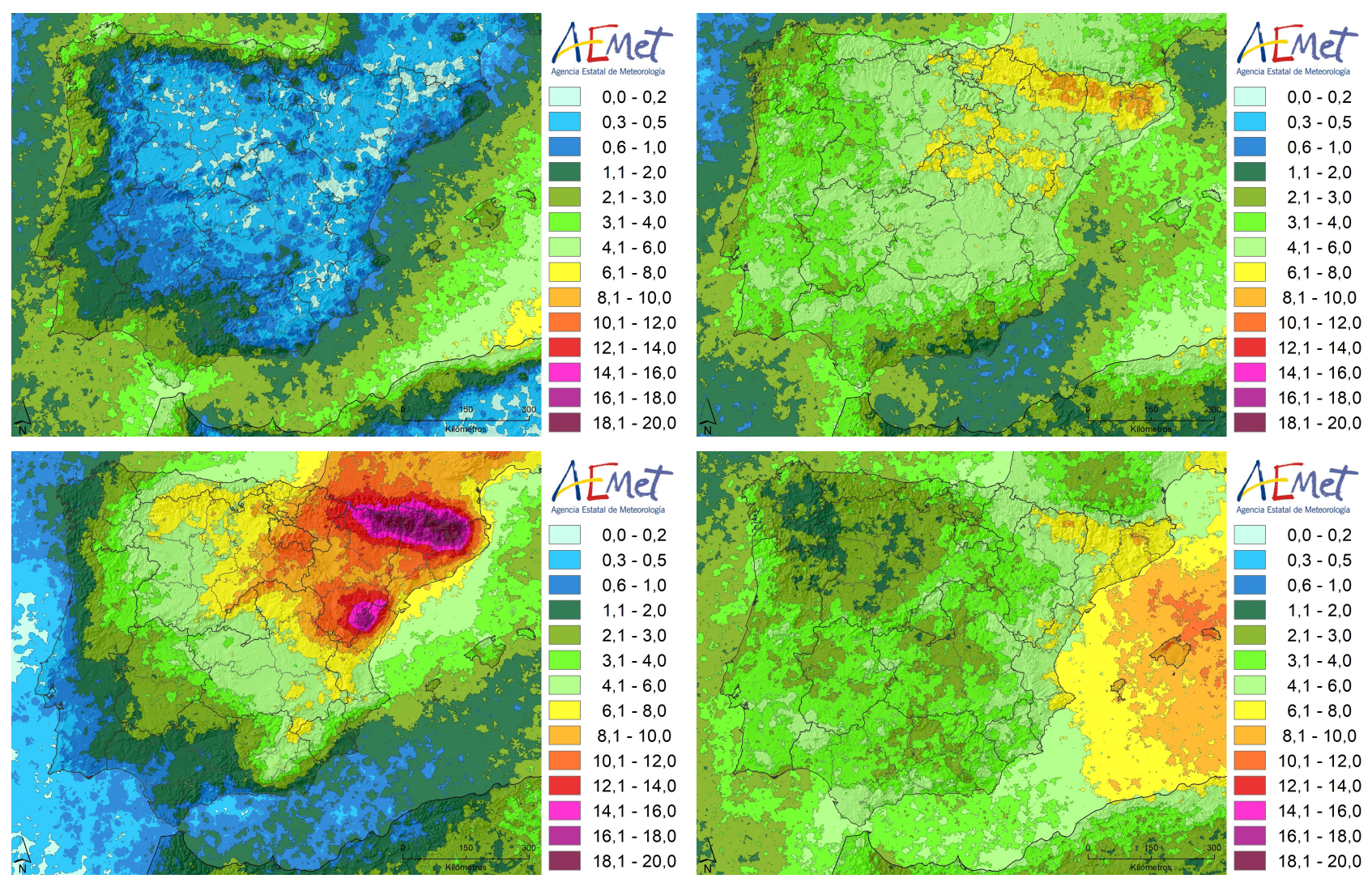

Figura 11. Número medio estacional de días de tormenta días de tormenta/trimestre). Arriba a la izquierda, invierno; arriba a la derecha, primavera; abajo a la izquierda, verano; abajo a la derecha, otoño. 
1 y 2 días, por lo que se observa un fuerte gradiente entre estas dos zonas. El contraste entre el mar Mediterráneo relativamente cálido y las primeras irrupciones de masas de aire fría son los ingredientes necesarios para la formación de tormentas en esta área durante esta época.

Por último es interesante disponer de una referencia, en el sentido comentado, de número medio de días de tormenta al año y por meses en las capitales de provincia y las ciudades autónomas. Esta referencia puede ser importante para diversos fines, climatológicos y en la predicción del tiempo. Se presenta una tabla ordenada por orden alfabético según el nombre de la ciudad (tabla 1). El código de colores empleado es el siguiente: en color azul se sombrea el mes con menor número de días de tormenta y en color rojo se sombrea el mes con mayor número de días de tormenta. Respecto al valor anual, se sombrean en colores cálidos las capitales con mayor número de días de tormenta y en colores fríos las capitales de provincia con menor número de días de tormenta.

\begin{tabular}{|c|c|c|c|c|c|c|c|c|c|c|c|c|c|}
\hline \multicolumn{14}{|c|}{$\begin{array}{l}\text { Estadística de días de tormenta en capitales de provincia } \\
\qquad \text { (periodo 2007-2016) }\end{array}$} \\
\hline Capital & ene. & feb. & mar. & abr. & may. & jun. & jul. & ago. & sep. & oct. & nov. & dic. & anual \\
\hline A Coruña & 1,4 & 1,4 & 0,6 & 0,8 & 0,8 & 0,4 & 0,2 & 0,6 & 0,4 & 0,4 & 1,8 & 0,7 & 9,5 \\
\hline Albacete & 0,1 & 0,1 & 0,3 & 1,6 & 2,4 & 2,6 & 1,1 & 1,7 & 2,2 & 1,1 & 0,6 & 0,1 & 13,9 \\
\hline Alicante/Alacant & 0,2 & 0,2 & 0,1 & 1,4 & 1,4 & 1,0 & 0,3 & 1,0 & 2,6 & 1,6 & 0,9 & 0,4 & 11,1 \\
\hline Almería & 0,1 & 0,1 & 0,6 & 0,4 & 0,5 & 0,7 & 0,1 & 0,4 & 1,2 & 0,9 & 0,7 & 0,6 & 6,3 \\
\hline Ávila & 0,0 & 0,1 & 0,4 & 1,5 & 2,6 & 2,0 & 2,1 & 2,0 & 2,3 & 0,7 & 0,1 & 0,0 & 13,8 \\
\hline Badajoz & 0,1 & 0,5 & 0,5 & 1,9 & 1,2 & 0,8 & 0,3 & 0,5 & 1,6 & 0,8 & 0,3 & 0,3 & 8,8 \\
\hline Barcelona & 0,4 & 0,3 & 0,8 & 2,1 & 2,3 & 2,3 & 2,9 & 3,0 & 2,9 & 2,8 & 1,4 & 0,4 & 21,6 \\
\hline Bilbao & 1,4 & 1,3 & 1,1 & 2,1 & 2,1 & 2,9 & 2,0 & 1,6 & 1,6 & 1,0 & 1,1 & 0,8 & 19,0 \\
\hline Burgos & 0,1 & 0,4 & 0,3 & 1,6 & 2,4 & 3,0 & 1,9 & 1,7 & 1,4 & 0,6 & 0,2 & 0,0 & 13,6 \\
\hline Cáceres & 0,0 & 0,3 & 0,7 & 2,1 & 1,1 & 1,1 & 0,6 & 1,1 & 2,1 & 0,5 & 0,2 & 0,4 & 10,2 \\
\hline Cádiz & 1,0 & 1,2 & 1,1 & 1,4 & 0,5 & 0,2 & 0,0 & 0,6 & 1,4 & 1,2 & 2,3 & 1,5 & 12,4 \\
\hline Castellón de la Plana/Castelló de la Plana & 0,2 & 0,1 & 0,3 & 1,5 & 2,4 & 2,9 & 1,6 & 2,7 & 3,1 & 1,5 & 0,9 & 0,5 & 17,7 \\
\hline Ceuta & 1,0 & 1,3 & 1,5 & 1,1 & 0,3 & 0,5 & 0,2 & 0,4 & 1,1 & 1,6 & 1,5 & 2,0 & 12,5 \\
\hline Ciudad Real & 0,0 & 0,2 & 0,3 & 1,9 & 2,1 & 2,1 & 1,5 & 1,4 & 2,6 & 0,9 & 0,4 & 0,4 & 13,8 \\
\hline Córdoba & 0,2 & 0,6 & 0,6 & 1,6 & 1,6 & 0,8 & 0,3 & 0,5 & 1,2 & 0,9 & 0,5 & 0,7 & 9,5 \\
\hline Cuenca & 0,1 & 0,0 & 0,2 & 2,5 & 2,6 & 3,0 & 2,1 & 2,1 & 2,6 & 0,7 & 0,1 & 0,1 & 16,1 \\
\hline Donostia/San Sebastián & 1,2 & 1,3 & 2,0 & 1,4 & 2,9 & 3,5 & 2,9 & 2,3 & 1,7 & 1,4 & 1,7 & 1,8 & 24,1 \\
\hline Girona & 0,6 & 0,8 & 1,2 & 2,2 & 3,4 & 3,4 & 3,5 & 3,9 & 3,4 & 2,2 & 1,5 & 0,2 & 26,3 \\
\hline Granada & 0,1 & 0,2 & 0,4 & 0,6 & 1,4 & 0,9 & 0,4 & 0,6 & 1,6 & 1,2 & 0,8 & 0,5 & 8,7 \\
\hline Guadalajara & 0,2 & 0,5 & 0,5 & 2,4 & 3,9 & 2,9 & 2,0 & 2,1 & 1,3 & 1,5 & 0,1 & 0,1 & 17,5 \\
\hline Huelva & 0,8 & 0,7 & 1,2 & 1,3 & 1,4 & 0,0 & 0,2 & 0,5 & 0,7 & 1,0 & 1,4 & 1,7 & 10,9 \\
\hline Huesca & 0,1 & 0,1 & 0,8 & 2,9 & 3,8 & 5,0 & 3,9 & 3,9 & 3,3 & 1,9 & 0,5 & 0,1 & 26,3 \\
\hline Jaén & 0,3 & 0,4 & 0,6 & 1,7 & 1,1 & 1,4 & 0,4 & 0,6 & 1,8 & 0,7 & 0,4 & 0,5 & 9,9 \\
\hline Las Palmas de Gran Canaria & 0,1 & 0,3 & 0,5 & 0,0 & 0,0 & 0,0 & 0,1 & 0,1 & 0,2 & 0,4 & 0,5 & 0,3 & 2,5 \\
\hline León & 0,1 & 0,1 & 0,1 & 1,8 & 2,7 & 2,4 & 1,9 & 1,8 & 1,4 & 0,5 & 0,2 & 0,1 & 13,1 \\
\hline Lleida & 0,2 & 0,3 & 0,3 & 1,8 & 2,0 & 3,5 & 3,3 & 3,0 & 3,0 & 1,9 & 0,6 & 0,0 & 19,9 \\
\hline Logroño & 0,1 & 0,2 & 0,6 & 1,9 & 2,5 & 3,0 & 3,6 & 2,2 & 1,8 & 0,4 & 0,3 & 0,0 & 16,6 \\
\hline Lugo & 0,4 & 0,1 & 0,1 & 0,9 & 1,5 & 1,1 & 0,7 & 0,9 & 0,9 & 0,3 & 0,3 & 0,2 & 7,4 \\
\hline Madrid & 0,0 & 0,4 & 0,2 & 1,6 & 2,9 & 2,0 & 1,6 & 1,5 & 1,7 & 0,9 & 0,3 & 0,0 & 13,1 \\
\hline Málaga & 0,6 & 1,0 & 0,6 & 0,9 & 0,7 & 0,4 & 0,3 & 0,3 & 1,5 & 1,5 & 1,0 & 0,9 & 9,7 \\
\hline Melilla & 1,6 & 0,6 & 0,6 & 0,4 & 0,3 & 0,4 & 0,4 & 0,3 & 1,0 & 1,2 & 1,5 & 0,9 & 9,2 \\
\hline Murcia & 0,1 & 0,1 & 0,7 & 1,2 & 1,5 & 1,1 & 0,2 & 0,6 & 1,4 & 1,2 & 0,5 & 0,2 & 8,8 \\
\hline Ourense & 0,4 & 0,4 & 0,2 & 2,2 & 1,4 & 1,1 & 1,0 & 0,7 & 1,0 & 0,4 & 0,4 & 0,2 & 9,4 \\
\hline Oviedo & 0,8 & 0,9 & 1,0 & 2,0 & 2,3 & 2,3 & 1,0 & 2,1 & 1,5 & 0,3 & 1,2 & 0,5 & 15,9 \\
\hline Palencia & 0,1 & 0,4 & 0,3 & 1,6 & 2,6 & 2,9 & 2,0 & 1,1 & 1,4 & 0,6 & 0,1 & 0,0 & 13,1 \\
\hline Palma & 0,9 & 1,1 & 0,8 & 0,6 & 1,9 & 1,0 & 0,2 & 1,7 & 3,9 & 2,5 & 2,9 & 1,0 & 18,5 \\
\hline Pamplona/Iruña & 0,4 & 0,5 & 1,3 & 2,5 & 3,1 & 3,6 & 3,7 & 3,3 & 2,6 & 1,6 & 0,3 & 0,1 & 23,0 \\
\hline Pontevedra & 1,8 & 1,0 & 0,7 & 1,1 & 0,9 & 0,8 & 0,5 & 0,5 & 0,5 & 1,0 & 1,7 & 0,7 & 11,2 \\
\hline Salamanca & 0,0 & 0,1 & 0,4 & 1,6 & 2,5 & 1,6 & 1,8 & 1,2 & 1,9 & 0,5 & 0,2 & 0,4 & 12,2 \\
\hline Santa Cruz de Tenerife & 0,3 & 0,8 & 0,7 & 0,0 & 0,0 & 0,0 & 0,0 & 0,3 & 0,0 & 0,2 & 0,4 & 0,4 & 3,1 \\
\hline Santander & 1,8 & 1,7 & 0,9 & 0,6 & 2,0 & 2,0 & 1,3 & 2,1 & 0,8 & 0,5 & 2,0 & 1,2 & 16,9 \\
\hline Segovia & 0,0 & 0,0 & 0,3 & 1,7 & 3,0 & 2,8 & 2,9 & 2,1 & 2,1 & 0,8 & 0,1 & 0,1 & 15,9 \\
\hline Sevilla & 0,3 & 0,6 & 0,9 & 1,6 & 1,0 & 0,2 & 0,3 & 0,5 & 1,5 & 1,4 & 1,2 & 1,6 & 11,1 \\
\hline Soria & 0,0 & 0,4 & 0,4 & 1,8 & 3,8 & 3,7 & 3,6 & 3,0 & 2,3 & 1,2 & 0,1 & 0,0 & 20,3 \\
\hline Tarragona & 0,4 & 0,3 & 0,5 & 1,5 & 1,9 & 2,6 & 2,6 & 2,9 & 3,3 & 2,6 & 1,4 & 0,5 & 20,5 \\
\hline Teruel & 0,0 & 0,0 & 0,4 & 1,6 & 3,0 & 4,3 & 3,9 & 3,8 & 2,9 & 0,8 & 0,0 & 0,1 & 20,8 \\
\hline Toledo & 0,0 & 0,2 & 0,5 & 1,8 & 2,4 & 2,6 & 1,3 & 1,4 & 1,8 & 1,2 & 0,2 & 0,2 & 13,6 \\
\hline València & 0,3 & 0,1 & 0,6 & 1,6 & 2,4 & 1,8 & 1,8 & 1,7 & 2,8 & 1,9 & 0,8 & 0,4 & 16,2 \\
\hline Valladolid & 0,0 & 0,1 & 0,5 & 1,4 & 2,1 & 2,5 & 1,8 & 1,6 & 2,0 & 0,4 & 0,1 & 0,0 & 12,5 \\
\hline Vitoria-Gasteiz & 0,2 & 0,4 & 1,0 & 2,5 & 2,9 & 3,2 & 2,6 & 1,8 & 1,7 & 1,0 & 0,3 & 0,2 & 17,8 \\
\hline Zamora & 0,1 & 0,1 & 0,6 & 1,2 & 2,0 & 1,6 & 1,2 & 1,3 & 1,8 & 0,5 & 0,3 & 0,0 & 10,7 \\
\hline Zaragoza & 0,2 & 0,1 & 0,3 & 2,0 & 2,8 & 3,6 & 3,1 & 3,6 & 2,1 & 1,4 & 0,3 & 0,0 & 19,5 \\
\hline
\end{tabular}

Tabla 1. Estadística de días de tormenta en las capitales de provincia y ciudades autónomas (ordenación alfabética). 


\section{NÚMERO DE DESCARGAS EN CADA DÍA DE TORMENTA}

Al igual que existía una gran variabilidad espacial y temporal en la distribución de la densidad de descargas eléctricas y del número de días de tormenta, también existe una gran variabilidad espacial y temporal en el número de descargas que generan las tormentas. Si en cada día de tormenta contamos el número de descargas en el promedio de la superficie del área de la Península, Baleares, Ceuta y Melilla (solo de aquellos lugares en los que haya habido tormenta) se registra una media de 13,8 descargas en cada día tormenta.

Las tormentas que más descargas generan son las que se registran en plena canícula, durante los últimos días de julio y los primeros de agosto, y las tormentas que menos generan son las de mitad del mes de febrero, entre el 14 y el 22 de este mes.

La zona de mayor densidad de descargas en cada día de tormenta se observa en el mar balear, entre la costa de Valencia, Castellón, Tarragona, Barcelona y las islas Baleares, penetrando la zona de máximos por la costa de estas provincias y extendiéndose hacia el interior por la provincia de Castellón hasta el Maestrazgo turolense.

Resulta obvio que las tormentas muy activas eléctricamente y a la vez estacionarias contribuyen de forma muy notable a los máximos de la figura 12. Por ello, una de las causas que podría justificar que el máximo de descargas en cada día de tormenta se encuentre en zonas marítimas mediterráneas, es porque resulta frecuente que la convección que se desarrolla en el Mediterráneo, sobre todo en otoño, lo haga en un ambiente de moderada o débil cizalladura, en las que el fuerte empuje hidrostático es el mecanismo predominante, lo que favorece que las células convectivas generadas en muchas ocasiones tengan un movimiento lento o incluso permanezcan estacionarias durante horas en una zona, en oposición a las tormentas de rápido movimiento en las que, aunque globalmente generan muchas descargas en el ciclo de vida de las mismas, particularmente no dejan muchas descargas alrededor de un observatorio por su rápido paso sobre el mismo. Por otro lado, y relacionado con el aspecto anterior, la frecuencia de sistemas convectivos de mesoescala en esta zona, que por su organización interna y persistencia son generadores de gran número de descargas eléctricas, contribuyen de forma determinante a la existencia de este máximo. Es precisamente esa zona del mar la que presenta una mayor temperatura superficial a finales de verano y durante gran parte del otoño.

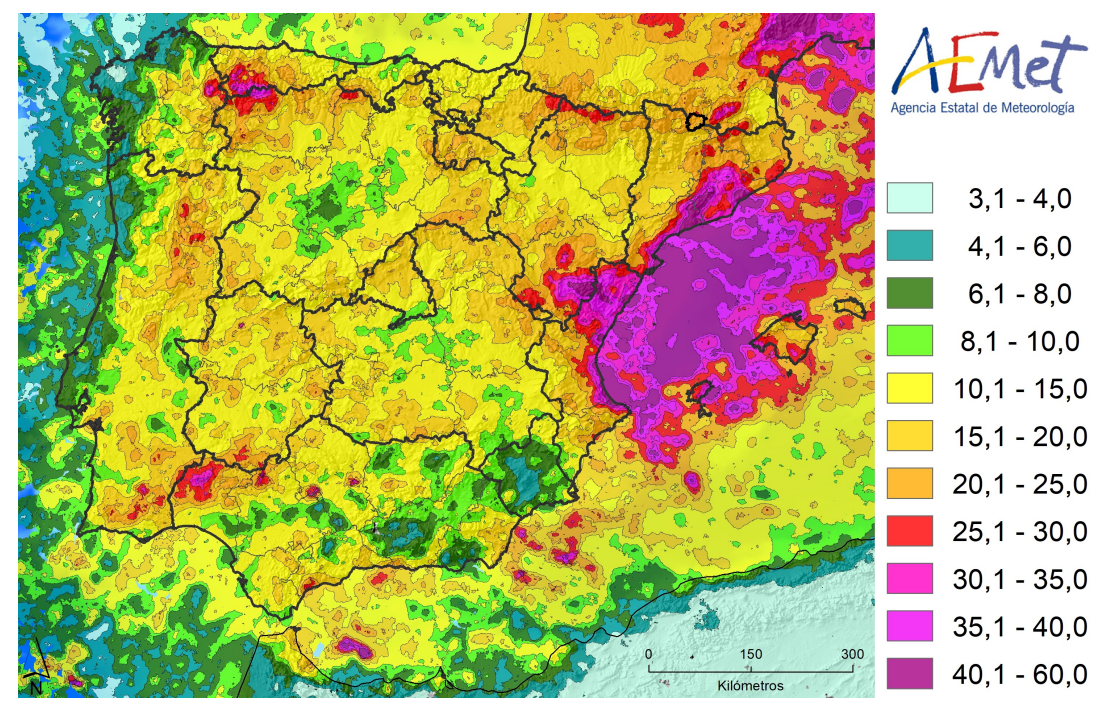

Figura 12. Número medio de descargas eléctricas en cada día de tormenta. 


\section{REFERENCIAS}

Changnon JR., S. A., 1989. Relations of Thunderstorms and Cloud-to-Ground Lightning Frequencies. Journal of Climate, 2 (8), 897-921. https://doi.org/10.1175/1520-0442(1989)002<0897:ROTACT>2.0.CO;2.

Czernecki, B., Taszarek, M., Kolendowicz, L. y Konarski, J., 2016. Relationship between human observations of thunderstorms and the PERUN lightning detection network in Poland. Atmospheric Research, 167, 118-128. https://doi.org/10.1016/j.atmosres.2015.08.003.

FInKE, U. y HAUf, T., 1996. The characteristics of lightning occurrence in southern Germany. Contributions to Atmospheric Physics.

Fleagle, R. G., 1949. The audibility of thunder. J. Acoust. Soc. Am., 21 (4), 411-412. http://dx.doi.org/ $10.1121 / 1.1906528$.

GómeZ ViñAs, P., 2016. Climatología de tormentas en Galicia a partir de datos de la red de detección de descargas eléctricas. Delegación Territorial de AEMET en Galicia (ACoruña).

Mäkelä, A., EnNo, S. E. y HaApalainen, J., 2014. Nordic Lightning Information System: Thunderstorm climate of Northern Europe for the period 2002-2011. Atmospheric Research, 139, 46-61. https://doi.org/ 10.1016/j.atmosres.2014.01.008.

Pérez Puebla, F., Zancajo Rodríguez, C. y González Márquez, J., 2007. Aproximaciones al concepto de tormenta eléctrica. Calendario meteorológico 2008. Instituto Nacional de Meteorología.

Sanz, R., Hernández, M., José, A., Przeor, M., González, P., Prats, N. y Barrera, E., 2016. Análisis de los rayos registrados en Canarias en el periodo 2006-2015. Calendario meteorológico 2017, 241-254. AEMET.

World Meteorological Organization, 1956. World Distribution of Thunderstorm Days. WMO/OMM, No. 21, TP 2, Geneva. 